\title{
Rehybridization of electronic structure in compressed two-dimensional quantum dot superlattices
}

\author{
G. Medeiros-Ribeiro, ${ }^{*}$ D. A. A. Ohlberg, and R. Stanley Williams \\ Hewlett-Packard Labs, 3500 Deer Creek Road, MS 26U-12, Palo Alto, California 94304-1392 \\ James R. Heath \\ Department of Chemistry and Biochemistry, University of California at Los Angeles, Los Angeles, California 90095-1569
}

(Received 8 October 1998)

\begin{abstract}
Two-dimensional superlattices of organically passivated 2.6-nm silver quantum dots were prepared as Langmuir monolayers and transferred to highly oriented pyrolytic graphite substrates. The structural and electronic properties of the films were probed with variable temperature scanning tunneling microscopy. Particles passivated with decanethiol (interparticle separation distance of $\sim 1.1 \pm 0.2 \mathrm{~nm}$ ) exhibited Coulomb blockade and staircase. For particles passivated with hexanethiol or pentanethiol (interparticle separation distance of $\sim 0.5$ $\pm 0.2 \mathrm{~nm}$ ), the single-electron charging was quenched, and the redistribution of the density of states revealed that strong quantum mechanical exchange, i.e., wave-function hybridization, existed among the particles in these films. [S0163-1829(99)03604-8]
\end{abstract}

Architectonic solids of quantum dots (QD's), or QD superlattices, represent a new class of materials for studying the fundamentals of charge transport. In any mechanism for the electrical conductivity of a solid, the key physical parameters are the site charging energy, the strength of coupling between the sites, and the symmetry of the solid. ${ }^{1-3}$ In principle, all three are chemically controllable in QD superlattices, and thus it should be possible to "engineer' the bulk electronic properties.

Superlattices of both metal ${ }^{4-6}$ and semiconductor ${ }^{7,8}$ QD's have been fabricated by several groups over the past few years. For two-dimensional (2D) superlattices, the site charging energies ${ }^{9}$ for hexagonal structures of organically passivated Ag QD's have been measured by capacitance spectroscopy. ${ }^{10}$ For those measurements, an active region of up to $10^{5}$ particles was incorporated into a single capacitor. Single-particle site charging energies, in the form of Coulomb blockade and staircase characteristics, were reported, and all observations were consistent with the standard model for size-dependent charging energies in a single metallic quantum dot. ${ }^{11}$

Site-site coupling in QD superlattices has been measured for both semiconductor and metallic QD's. For the case of semiconductor QD-based solids, classical coupling between dots, manifested as Forster, or dipole coupling, was observed, ${ }^{12}$ but quantum mechanical coupling was not documented. For the case of 2D solids made from metal QD's, effects from both classical and quantum coupling have been recently reported. ${ }^{13,14}$ In those experiments, the interparticle separation distance between organically passivated silver QD's was varied continuously from $\delta=2.0 \mathrm{~nm}$ to $\delta$ $=0.4 \mathrm{~nm}$ (where $\delta$ equals the distance between the surfaces of the metallic cores of the nanocrystals). For $2.0 \mathrm{~nm}<\delta$ $<1.0 \mathrm{~nm}$, interparticle coupling could be accounted for with simple classical dipole-based models. ${ }^{14}$ For $1.0 \mathrm{~nm}<\delta$ $<0.5 \mathrm{~nm}$, various manifestations of quantum coupling were observed in the linear and nonlinear optical responses of the superlattices. Around $\delta \sim 0.5 \mathrm{~nm}$, a metal/insulator transition was observed both optically ${ }^{13}$ and through electron transport measurements. ${ }^{15}$ Such a transition represents the "extreme" case of quantum coupling: The individual electronic identity of the nanocrystals was lost, and the superlattices resembled bulk, metallic films. All of these phenomena were reversible. The interparticle separation distance was controlled with a Langmuir trough. ${ }^{16}$ If a monolayer of nanocrystals was compressed through the MI transition, then the transition could be reversed either by expanding the barriers, or by redissolving the nanocrystals as a colloid. Thus, the chemical identity of the particles was retained in these experiments.

In this paper, we utilize variable temperature scanning tunnel microscopy (STM) to characterize 2D Ag QD superlattices that have been similarly prepared on a Langmuir trough and subsequently transferred to highly oriented pyrolytic graphite (HOPG). Graphite is a zero-gap semiconductor with weak electronic coupling normal to the carbon planes. Also, the graphite work function is at least $0.5 \mathrm{eV}$ larger than that of the Ag dots. Thus, the contact potential and the mismatches in the density of states and the orbital symmetry effectively isolate the Ag dots from the substrate.

We have carried out a series of experiments aimed at understanding how the electronic signatures of individual particles are modified as a function of interparticle separation distance. An isolated particle, as well as ordered hexagonal monolayers of $2.6 \mathrm{~nm}$ diameter silver QD's, capped with decanethiol $\left(\mathrm{C}_{10}\right)$, hexanethiol $\left(\mathrm{C}_{6}\right)$, and pentanethiol $\left(\mathrm{C}_{5}\right)$, were investigated. For the films, we found that $2 \mathrm{D}$ superlattice domain sizes of at least $100 \mathrm{~nm}$ lateral dimension ( $\sim 500$ particles) were readily achieved. The current-voltage $(I-V)$ spectra of isolated particles and superlattices of $\mathrm{C}_{10}$ particles $(\delta=1.1 \pm 0.2 \mathrm{~nm})$ displayed single-electron charging in the form of Coulomb blockade and staircase structure. Both $\mathrm{C}_{6}$ and $\mathrm{C}_{5}$ monolayers $(\delta=0.5 \pm 0.2 \mathrm{~nm})$ were characterized by very different $I-V$ curves with no observable charging phenomena, and exhibited a temperature independent, finite density of states at $0 \mathrm{~V}$.

The ease in imaging the monolayers was related to the length of the passivating organic group: same-sized metal 


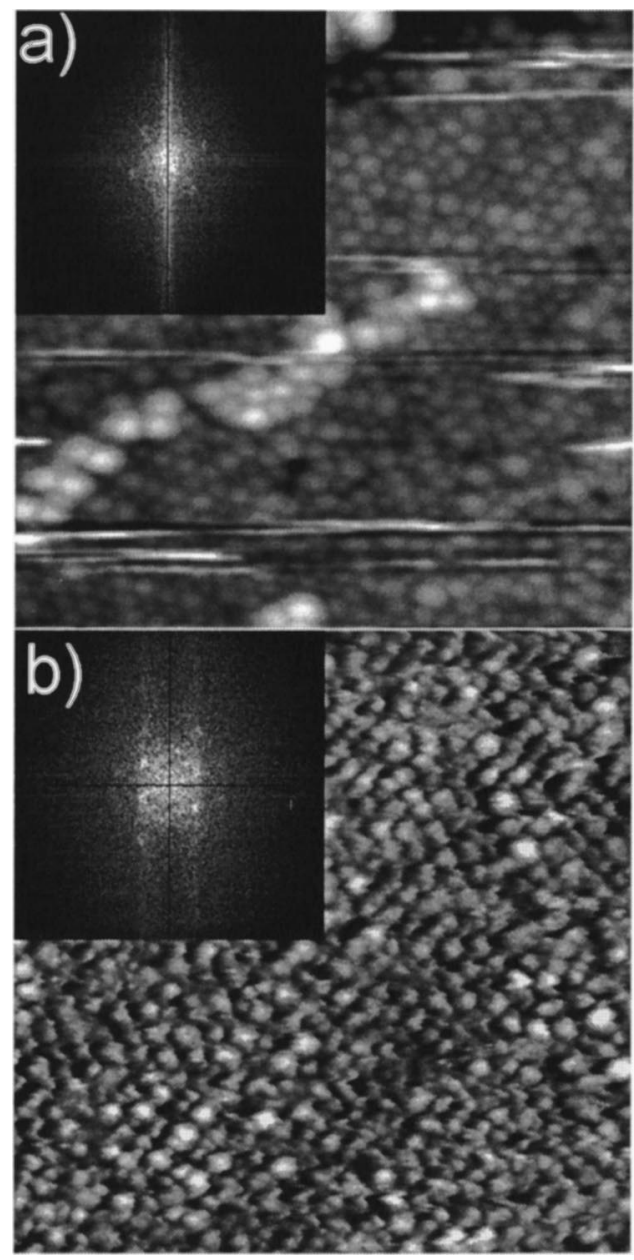

FIG. 1. $100 \times 100 \mathrm{~nm}^{2}$ STM micrographs of hexanethiolcapped (a) and decanethiol-capped (b) 2.6-nm-diameter silver quantum dot monolayers after transfer to a HOPG substrate. The Fourier transform of these images is presented at the top left. The interdot separation distances are $\sim 0.5 \mathrm{~nm}$ for the hexanethiol capped particles, and $\sim 1.1 \mathrm{~nm}$ for the decanethiol capped particles.

cores with shorter ligands were characterized by stronger interparticle dispersion attractions. ${ }^{4} \mathrm{C}_{10}$-particle monolayers were difficult to image at $300 \mathrm{~K}$, but were readily imaged at cryogenic temperatures $(<100 \mathrm{~K})$. Monolayers of $\mathrm{C}_{6}$ and $\mathrm{C}_{5}$ particles were readily imaged at all temperatures. In Fig. 1(a), we show an STM micrograph of a monolayer of $\mathrm{C}_{6}$ particles, collected at $20 \mathrm{~K}$. Figure $1(\mathrm{~b})$ is a $100 \times 100 \mathrm{~nm}^{2}$ STM scan of a supported monolayer of $\mathrm{C}_{10}$ particles, imaged at $100 \mathrm{~K}$. Images of $\mathrm{C}_{10}$ monolayers, coupled with previous TEM measurements, indicate particles with a 2.6-nmdiameter metal core, separated from their nearest neighbors by $1.1 \mathrm{~nm} . \mathrm{C}_{6}$ particles appear better defined, indicating a more rigid film.

Although the individual nanocrystals investigated in this study are metallic spheres (the discrete energy levels are only a few meV apart), ${ }^{17}$ the superlattices with interparticle separation distances $>1 \mathrm{~nm}$ are insulating. Within an extended metallic solid, the site charging energy is 0 . However, our "lattice sites" are the Ag QD's, which are characterized by a finite capacitance that generates a site charging energy of $0.34 \mathrm{eV}$. Thus, the superlattice is an insulator with a Coulombic band gap, while the individual particles that comprise

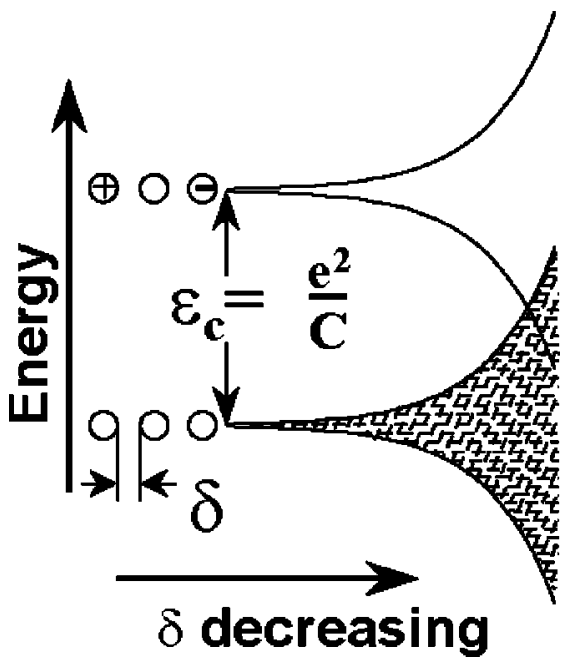

FIG. 2. Schematic many-electron diagram of the effects of interparticle separation distance $(\delta)$ on the electronic structure of the QD superlattice. The lower (cross-hatched) level represents the ground state of the system of dots, whereas the upper level is the excited state formed by introduction of an electron and a hole into the system. The charging energy of a single isolated particle is $e^{2} / 2 C$, where $C$ is the capacitance of the particle $\left(\sim 10^{-19} F\right)$. The energy to separate a positive and negative charge carrier is the width of the Coulomb gap [Fig. 3(a)], and is equal to $338 \mathrm{meV}$. As the lattice is compressed, quantum exchange interactions lead to the development of inter-dot energy bands, eventually leading to the formation of a metallic solid (where the cross-hatched and unshaded regions intersect).

it are metallic. However, as the interparticle separation in the superlattice is reduced, quantum interactions lead to the development of interdot energy bands with a finite width, and the Coulomb gap decreases, as shown schematically in Fig. 2. At some interparticle separation, the Coulomb gap disappears, and the system becomes metallic. ${ }^{18}$ Previous experiments ${ }^{13,15}$ indicated that such a transition for 3-nmdiameter Ag particles occurred at a $\delta$ of $0.5 \pm 0.2 \mathrm{~nm}$. Films made from same-sized particles at different interparticle separation distances should be characterized by different amounts of exchange coupling.

Current imaging tunneling spectroscopy (CITS) images were collected on $50 \times 50 \mathrm{~nm}^{2}$ areas in $32 \times 32$ arrays. The $I-V$ curves were averaged over individual QD's and entire images, to investigate the statistical properties of the films. As pointed out recently, ${ }^{19} I-V$ measurements of metal nanoparticles with an STM depend quite significantly upon the exact position of the tip with respect to the particle. Although the monolayers were characterized by substantial regularity in both particle size and packing density, the $I-V$ curves for the $\mathrm{C}_{10}$ particles varied considerably as a function of position and temperature. $I-V$ curves for the $\mathrm{C}_{6}$ and $\mathrm{C}_{5}$ particles were essentially independent of position and temperature. For all particles, certain observations were generally reproducible. In particular, the nanocrystal packing density was found to strongly influence the single-particle $I-V$ curves, and the $\mathrm{C}_{10}$ particle monolayer $I-V$ curves consistently exhibited richer spectra than was observed for the isolated particle or the $\mathrm{C}_{6}$ and $\mathrm{C}_{5}$ monolayers. Finally, after completion of a set of experiments on $\mathrm{C}_{10}$ particles, the STM tip was scanned over a clean HOPG surface. The tip had apparently "picked up" a 


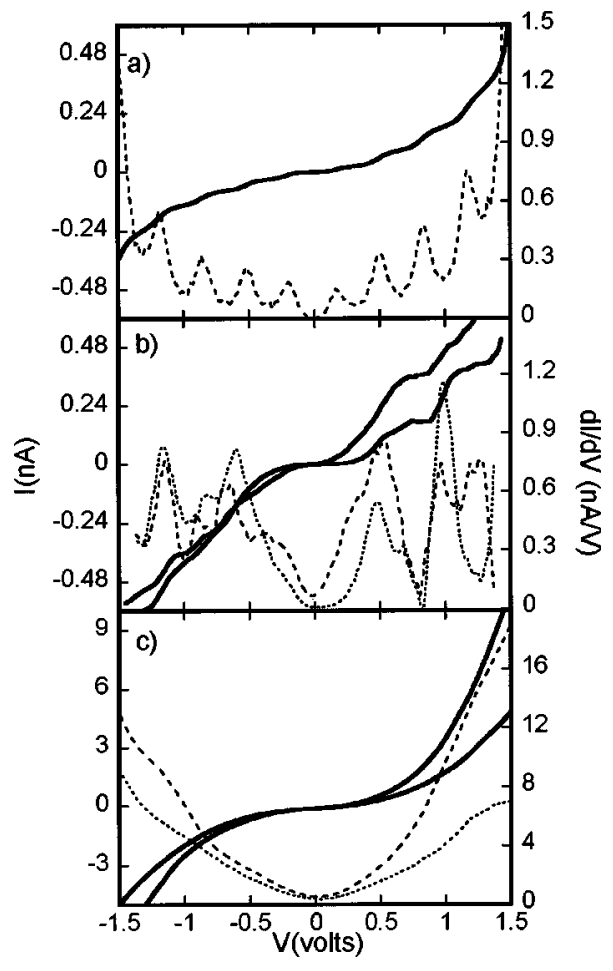

FIG. 3. The effect of interparticle separation distance on the single-particle charging states within a 2D QD superlattice is presented (solid lines, $I-V$; dashed lines $d I / d V$ ). (a) An $I-V$ measurement and its derivative for an isolated decanethiol capped 2.6-nm diameter Ag quantum dot. Analysis of the blockade and staircase structure indicates a charging energy of $338 \mathrm{meV}$. (b) Two representative $I-V$ measurements and their derivatives for the decanethiol QD monolayer imaged in Fig. 1(b), collected at $20 \mathrm{~K}$. Notice that, although the structure of the curves varies substantially from that of an isolated particle, single-particle charging effects are present. (c) Two representative $I-V$ measurements and their derivatives for the hexanethiol capped QD monolayer imaged in Fig. 1(a). Notice that all signs of single-electron charging effects are gone. $V_{\text {gap }}=-1 \mathrm{~V} ; I_{\text {tunn }}=0.02,0.02$, and $0.1 \mathrm{nA}$ for $(\mathrm{a}),(\mathrm{b})$, and (c), respectively.

particle, and thus the $I-V$ curve of an isolated particle was recorded. This curve was recorded several times in between when the tip was moved around the HOPG surface, and, until the tip was cleaned, the measured $I-V$ curve did not change.

In Figs. 3(a)-3(c), we show $I-V$ curves for the isolated $\mathrm{C}_{10}$ particle, and for the $\mathrm{C}_{10}$ and $\mathrm{C}_{6}$ Ag quantum dot monolayers, respectively. For the isolated particle, we measure a nearly textbook Coulomb blockade and staircase structure. Similar data have also been reported by two groups ${ }^{22,23}$ for $\mathrm{Au}$ particles on $\mathrm{Au}$ surfaces with dielectric layers corresponding to our $\mathrm{C}_{6}$ ligands. For the $\mathrm{C}_{10}$ particle monolayer, we see an asymmetric Coulomb blockade and an irregular staircaselike structure. The measured structure is distinct from that observed for the isolated particle. Although we do not yet understand all the details of the staircase, we find that the Coulomb-blockade regions can be classified according to the schemes proposed by Hanna and Tinkham for single QD's. ${ }^{20}$ The implication is that in these $\mathrm{C}_{10}$ monolayers, the particles individually have capacitances on the order of 1 $-2 \times 10^{-19} \mathrm{~F}$, and the measured circuit includes two or more

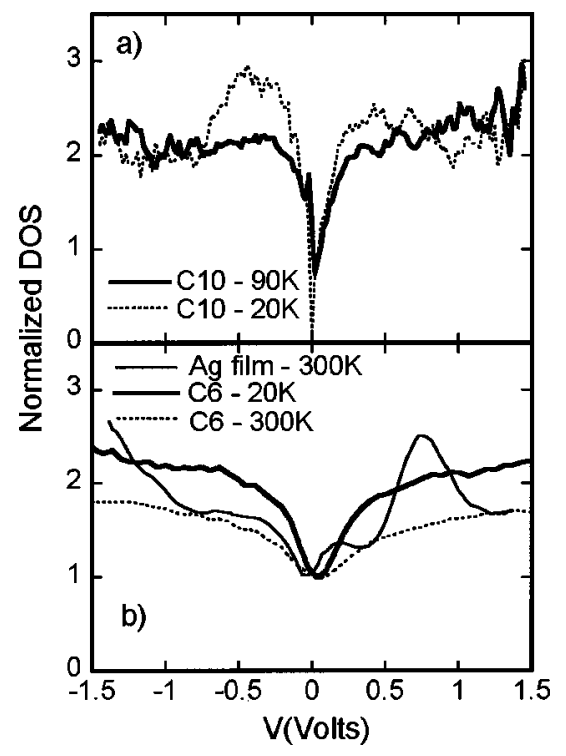

FIG. 4. Normalized density of states measurements for the decanethiol passivated QD's (a) and for the hexanethiol passivated QD's (b), as a function of temperature. $k T / q$ at 20 and $90 \mathrm{~K}$ is respectively, 1.7 and $7.8 \mathrm{meV}$. The DOS exhibits a strong temperature dependence near $0 \mathrm{~V}$ for the decanethiol capped particles, consistent with the presence of a Coulomb gap. In the bottom image, the DOS at $0 \mathrm{~V}$ is temperature independent throughout the range 300 to $20 \mathrm{~K}$, and is similar to that observed for a thin evaporated $\mathrm{Ag}$ film. This measurement is consistent with the notion that the hexanethiol capped QD monolayer is metallic. Notice that the evaporated film has a surface state near 0.2 and $0.8 \mathrm{eV}$, which is absent from the organically passivated particles.

of these particles. ${ }^{21}$ For the $\mathrm{C}_{6}$ particles, we see monotonic $I-V$ characteristics, and some slight asymmetry. For this system, we did not see any significant differences between $I-V$ curves collected over individual particles and ensemble averages, regardless of temperature.

We also present the normalized conductances, $(d I / d V)(V / I)$, for the two QD monolayers: 90- and 20-K data for $\mathrm{C}_{10} \mathrm{Ag}$ QD's in Fig. 4(a) and room temperature and $20 \mathrm{~K}$ data for $\mathrm{C}_{6} \mathrm{Ag}$ QD's in Fig. 4(b). If the normalized conductances are collected at sufficiently low biases, they are good approximations to the density of states (DOS) of a system. Semiconductors and insulators are characterized by a vanishing DOS at $0 \mathrm{~V}$. In this region CITS does not obtain reliable information for wide band-gap materials. At $0 \mathrm{~V}$, metallic surfaces have a finite density of states, and by definition, the normalized conductance is identical to 1 .

In Fig. 4(a), we present the normalized conductance characteristics for the $\mathrm{C}_{10}$ QD monolayer at 90 and $20 \mathrm{~K}$. Note that $(d I / d V)(V / I) \rightarrow 0$ at $0 \mathrm{~V}$ as we cool the sample. This is a necessary condition for any insulator, but the strong temperature dependence also highlights the dominant role that (thermally activated) phonon-mediated hopping plays in charge transport at finite temperature. This is consistent with a recent report of lateral transport measurements through a 2D array of similarly passivated Au quantum dots. ${ }^{14}$ We also observe some periodic structure at both 90 and $20 \mathrm{~K}$ for positive energies (electrons tunneling from the tip to the sample), that are, more or less, equally spaced by $200 \mathrm{meV}$.

We observe in Fig. 4(b) a value of 1 at $0 \mathrm{~V}$ for the nor- 
malized conductance of the $\mathrm{C}_{6}$ QD monolayer, which is temperature independent from 300 to $20 \mathrm{~K}$. The DOS is symmetric at room temperature, although it appears slightly asymmetric at lower temperatures. Comparing the two systems, we note that the changes in the DOS are more abrupt and occur at lower energies for the $\mathrm{C}_{10}$ particles. These changes are smoothed out when particles get closer. This redistribution of states should stem from quantum-exchange interactions. For comparison, we measured the normalized conductance of a thin Ag film evaporated onto HOPG. Except for the surface states near 0.2 and $0.8 \mathrm{~V}$ observed in the evaporated film, the measured DOS was quite similiar to the one measured on the 2D superlattice, indicating metallic behavior in the QD monolayer.

We have investigated the temperature-dependent electronic signatures of individual metal quantum dots that comprise a $2 \mathrm{D}$ hexagonal superlattice. We find that the current- voltage characteristics of the individual dots are strongly dependent upon the interparticle separation distance. For dots that are separated by $\sim 1.0 \mathrm{~nm}$, we observe Coulomb blockade and staircase structure, and DOS measurements indicate that the 2D lattice is insulating at low temperatures. For dots that are separated by sufficiently short distances $(\sim 0.5 \pm 0.2 \mathrm{~nm})$, the signatures of individual quantum dots disappear, and are replaced with the electronic behavior of a metallic film. These dc measurements confirm that the ac behavior observed previously for AG dot superlattices is indeed caused by a metal-insulator transition.

We acknowledge Alex Bratkovski and Andrew Briggs for stimulating discussions. J.R.H. acknowledges the Packard Foundation and the Sloan Foundation for financial support, as well the Hewlett Packard Corporation and an NSFGOALI grant.
*Electronic address: medeiros@hpl.hp.com

†Electronic address: heath@ chem.ucla.edu

${ }^{1}$ L. P. Kouwenhoven, F. W. J. Hekking, B. J. van Wees, C. J. P. M. Harmans, C. E. Timmering, and C. T. Foxon, Phys. Rev. Lett. 65, 361 (1990).

${ }^{2}$ C. I. Duruoz, R. M. Clarke, C. M. Marcus, and J. S. Harris, Jr., Phys. Rev. Lett. 74, 3237 (1995).

${ }^{3}$ A. J. Rimberg, T. R. Ho, and John Clarke, Phys. Rev. Lett. 74, 4714 (1995).

${ }^{4}$ P. Ohara, Daniel V. Leff, James R. Heath, and William M. Gelbart, Phys. Rev. Lett. 75, 3466 (1995).

${ }^{5}$ A. Taleb, C. Petit, and M. P. Pileni, Chem. Mater. 9, 950 (1997).

${ }^{6}$ R. L. Whetten, J. T. Khoury, M. M. Alvarez, S. Murthy, I. Vezmar, Z. L. Wang, P. W. Stephens, C. L. Cleveland, W. D. Luedtke, and U. Landman, Adv. Mater. 8, 428 (1996)

${ }^{7}$ N. Herron, J. C. Calabrese, W. E. Farneth, and Y. Wang, Science 259, 1426 (1993).

${ }^{8}$ C. B. Murray, C. R. Kagan, and M. G. Bawendi, Science 270, 1335 (1995).

${ }^{9}$ Single Charge Tunneling, edited by H. Grabert and M. H. Devoret (Plenum, New York, 1992).

${ }^{10}$ G. Markovich, Daniel V. Leff, Sung-Wook Chung, Hermes M. Soyez, Bruce Dunn, and James R. Heath, Appl. Phys. Lett. 70, 3107 (1997); G. Markovich, T. Vossmeyer, E. DeIonno, and J. R. Heath, in Nanostructured Materials, edited by V. Shalaev and M. Moskovits, ACS Symposia Series No. 679 (ACS Books, Washington, D.C., 1997), Chap. 1.

${ }^{11}$ J. Lambe and R. C. Jaklevic, Phys. Rev. Lett. 22, 1371 (1969).
${ }^{12}$ C. R. Kagan, C. B. Murray, M. Nirmal, and M. G. Bawendi, Phys. Rev. Lett. 76, 1517 (1996); C. R. Kagan, C. B. Murray, and M. G. Bawendi, Phys. Rev. B 65, 8633 (1996).

${ }^{13}$ C. P. Collier, R. J. Saykally, J. J. Shiang, S. E. Henrichs, and J. R. Heath, Science 277, 1978 (1997).

${ }^{14}$ J. J. Shiang, J. R. Heath, C. P. Collier, and R. J. Saykally, J. Phys. Chem. B 102, 3425 (1998).

${ }^{15}$ G. Markovich, C. P. Collier, and J. R. Heath, Phys. Rev. Lett. 80, 3807 (1998).

${ }^{16}$ The preparation of both the particles and the Langmuir monolayers is described in J. R. Heath, C. M. Knobler, and D. V. Leff, J. Phys. Chem. B 101, 198 (1997).

${ }^{17}$ R. Kubo, J. Phys. Soc. Jpn. 17, 975 (1962).

${ }^{18}$ C. A. Stafford and S. Das Sarma, Phys. Rev. Lett. 72, 3590 (1994); R. Kotlyar and S. Das Sarma, Superlattices Microstruct. 20, 641 (1996).

${ }^{19}$ Kang-Ho Park, Jeong Sook Ha, Wan Soo Yun, Mincheol Shin, Kyoung-Wan Park, and El-Hang Lee, Appl. Phys. Lett. 71, 1469 (1997).

${ }^{20}$ A. E. Hanna and M. Tinkham, Phys. Rev. B 44, 5919 (1991).

${ }^{21}$ M. Amman, S. B. Field, and R. C. Jaklevic, Phys. Rev. B 43, 1146 (1991).

${ }^{22}$ Ronald P. Andres, Thomas Bein, Matt Dorogi, Sue Feng, Jason I. Henderson, Clifford P. Kubiak, William Mahoney, Richard G. Osifchin, and R. Reifenberger, Science 272, 1323 (1996).

${ }^{23}$ R. S. Ingram, M. J. Hostetler, R. W. Murray, T. G. Schaaff, J. Khoury, R. L. Whetten, T. P. Bigioni, D. K. Guthrie, and P. N. First, J. Am. Chem. Soc. 119, 9279 (1997). 